\title{
IDENTIDAD Y MEMORIA GENEALÓGICA. UNA APORTACIÓN AL ESTUDIO DE LA ANTROPONIMIA MEDIEVAL GALLEGA
}

\author{
IDENTITY AND GENEALOGICAL MEMORY. \\ AN APPROXIMATION TO THE STUDY OF \\ GALICIAN MEDIEVAL ANTHROPONOMY
}

\author{
EDUARDO PARDO DE GUEVARA Y VALDÉS \\ Instituto de Estudios Gallegos "P. Sarmiento" \\ CSIC, Santiago de Compostela
}

\begin{abstract}
Resumen: El conocimiento de la estructura interna -genealógica- de los linajes medievales permite observar al detalle los sistemas de identificación y, al tiempo, calibrar su protagonismo en el proceso de cristalización de aquéllos. En este artículo se ejemplifican algunos de sus perfiles más significativos a partir del caso gallego, como la importancia de las distintas costumbres onomásticas en la articulación y cohesión interna de las estirpes de la nobleza bajomedieval, así como en la conformación de la memoria genealógica o en su propia afirmación ante la sociedad.
\end{abstract}

Palabras clave: Repertorios onomásticos; Antropónimo; Patronímico y renombre; Afirmación social y memoria genealógica; nobleza gallega.

\begin{abstract}
Knowledge of the internal structure of medieval lineage makes the detailed observation of identification methods possible. It also measures its prominent role in their long term crystallization. Some of the most significant traits of the genealogical knowledge of medieval lineage based on the Galician case are exemplified in this article. Mainly: the importance of the different onomastic traditions in the organization and cohesion of the low medieval nobility's lineage, in the making of a genealogical record, or in its own affirmation towards society.
\end{abstract}

Keywords: Onomastic repertoire; Anthroponym; Patronymic; Renown or renowned; Social affirmation; Genealogical report; Galician nobility.

\section{SUMARIO}

Los repertorios onomásticos familiares. Del antropónimo regular a la asociación del patronímico.- La afirmación del linaje. El renombre y la transmisión de los alcumes.- Una huella cultural. El influjo de la materia de Bretaña en Galicia.

El estudio de los sistemas de identificación, las formas de nombrarse o distinguirse unos de otros, y su natural evolución son instrumentos valiosos para el historiador, particularmente cuando se desea estudiar los grupos sociales y las estructuras familiares. Por fortuna, los estudios de antroponimia han alcanzado, al impulso de la historiografía francesa, un relativo desarrollo en estos últimos años; en este contexto se sitúan algunas aportaciones parciales 
en relación con Galicia ${ }^{1}$. Sin embargo, es mucho todavía lo que deberá avanzarse para valorar con carácter global la antroponimia hispánica medieval y sus complejas implicaciones sociales.

En Galicia, como en el resto del occidente europeo, la costumbre seguida para distinguirse unos de otros consistió, en el periodo anterior al año mil, en el uso de un solo nombre o denominación, elegido entre un repertorio amplio y variado, aunque a veces se utilizara también una suerte de segundo nombre, cognomento, introducido con la fórmula qui vocant ..., o similar. Al avanzar el siglo XI comenzaron a ser frecuentes ya las referencias de filiación - en genitivo normalmente, aunque a veces también en nominativo-, a modo de designación complementaria. Esta fórmula nueva alcanzó un predominio absoluto al avanzar el siglo XII, particularmente entre la aristocracia, no tardando en alcanzar cierta presencia otras formas antroponímicas. En medio de esta diversificación se consolidaron los denominaciones de dos elementos, reservándose el segundo para aludir a funciones u oficios, caracteres físicos o procedencia geográfica, lo que sirvió inicialmente para identificar a ciertos individuos cuando sus nombres se repetían en el seno de una misma comunidad. Con respecto a estas nuevas fórmulas importa destacar que su uso tuvo una clara significación social, al menos durante los primeros momentos - siglos X y XI-, puesto que el nomem paternum fue característico sólo de los sectores más favorecidos de la sociedad laica; esto es, de los designados colectivamente como filii bonorum o filii bene nadorum. La creciente fosilización de esta costumbre, ya desde los comienzos del XIII, o acaso desde un poco antes, dio paso por fin a unos sistemas de denominación mucho más complejos.

Pero lo que interesa al objeto de estas páginas no es exactamente la descripción y detalle de estos sistemas de identificación, sino su propio protagonismo en la definitiva cristalización de los linajes y, muy particularmente, en el paulatino desarrollo de algunos de sus rasgos definitorios. De ahí, pues, mi interés en poner de relieve e ilustrar debidamente su carácter propio como cauce para la articulación de la cohesión interna de aquéllos y, no menos, para la perpetuación de la memoria y la propia afirmación social

${ }^{1}$ Abrió el camino E. RIVAS, Onomástica persoal do noroeste hispano, Lugo, 1991. Le siguieron después los sucesivos estudios de A.I. BOULLON AGRELO, precedidos por su tesis doctoral Contribución ó estudio da antroponimia medieval galega. Véanse además, por ejemplo, M.C. PALLARES y E. PORTELA, El sistema antroponímico de las mujeres en la Galicia medieval. Tumbos de Sobrado, en X. Castro y J. de Juana (dir. de edición), A muller na Historia de Galicia, IX Xornadas de Historia de Galicia, Ourense, 1995, pp. 45-65, y las diferentes aproximaciones incluidas en el volumen colectivo, coordinado por P. Martínez Sopena, Antroponimia y sociedad. Sistemas de identificación hispano-cristianos en los siglos IX a XIII, Santiago-Valladolid, 1995 ; por ejemplo, M.C. PALLARES y E. PORTELA, El sistema antroponímico en Galicia. Tumbos del monasterio de Sobrado. Siglos IX a XIII, pp. 21-47, y M. GoNZÁLEZ VÁZQUEZ Y F.J. PÉREZ RODRÍGUEZ, El sistema antroponímico en Galicia. Tumbo del monasterio de Samos. Siglos VIII al XII, pp. 49-71, M. DURANY y M.C. RODRÍGUEZ, El sistema antroponímico en El Bierzo. Tumbo de San Pedro de Montes. Siglos IX al XIII, pp. 73-102, o P. MARTÍNEZ SOPENA, La antroponimia leonesa. Un estudio del Archivo Catedral de León '(8761200) pp. 155-180. A estas selectas referencias incorporo, asimismo, la reciente aportación de J. DE SALAZAR ACHA, La utilidad de la genealogía para el conocimiento de la evolución de los usos onomásticos, en "Actas de las Jornadas de Onomástica de la Sociedad de Estudios Históricos de Navarra", Pamplona, 2005, pp. 177-197. 
del grupo familiar. Ocurrió así, como se verá a continuación, con los antropónimos o nombres de pila de uso regular, con las asociaciones de estos con los patronímicos, con la fosilización de aquéllas y de estos últimos y, naturalmente, ya por fin, con la generalización de los renombres o apellidos, entendidos ya propiamente como nombres de familia.

En este contexto y sólo como simple orientación me parece interesante avanzar antes algunas cuantificaciones indicativas para resaltar las preferencias durante el periodo de referencia; esto es, desde el acceso al trono de Enrique II (1369) hasta las decisivas cortes de Toledo, donde los Reyes Católicos fijaron las grandes líneas de su futura acción de gobierno $(1480)^{2}$. Sobre una base de datos formada por 6.400 entradas y a partir de un conjunto de 248 antropónimos, en cuyo cómputo se incluyen las más diversas variantes que se consignan en la documentación vaciada, se alcanzan los siguientes resultados:

Antropónimo

Juan

Pedro

Fernando

Ruy

Alfonso

Álvaro

Lope

\section{Entradas}

542

515

$427^{3}$

$408^{4}$

$345^{5}$

$233^{6}$

$233^{7}$

A cierta distancia se sitúan otros antropónimos de uso también ciertamente frecuente en el período de referencia, como los de Diego con 176 entradas, García con $171^{8}$, Vasco con $159^{9}$, Gómez con $130^{10}$ o Ares $113^{11}$. Y por debajo ya del centenar de entradas se sitúan otros muchos antropónimos; así, en orden decreciente, los de Martín con 96 entradas $^{12}$, Payo y Esteban

${ }^{2}$ Los datos se extraen del proyecto de investigación Diccionario biográfico de la Galicia de los Trastámara (1369-1480), desarrollado bajo la dirección del autor en el Instituto de Estudios Gallegos "Padre Sarmiento" con financiaciốn de la Fundación Pedro Barrié de la Maza. Los trabajos se iniciaron en el año 2000, sobre la base de casi dos décadas de investigaciones previas, y todavía están sin concluir. De ahí, pues, que las cuantificaciones ofrecidas tengan sólo un valor relativo, a modo de simple orientación, particularmente a causa de las frecuentes homonimias, que en este momento se encuentran en proceso de revisión.

${ }^{3}$ De ellas, 155 remiten a la forma propia de Fernán y una sola a la de Ferrán.

${ }^{4}$ De ellas, 156 remiten a la forma castellana de Rodrigo y 40 a la más propia de Roy.

${ }^{5}$ De ellas, 27 remiten a la forma más propia de Alonso.

${ }^{6}$ De ellas, 10 remiten a la forma propia de Álvar.

${ }^{7}$ De ellas, 40 remiten a la forma propia de Lopo.

${ }^{8}$ De ellas, sólo cuatro remiten a otras formas arcaicas, como Garcie, Garsia o Garssie.

${ }^{9}$ De ellas, sólo una remite a la forma Velasco.

${ }^{10}$ De ellas, sólo dos remiten a la forma Gomecius.

${ }^{11}$ De ellas, 27 remiten a la forma castellana de Arias y sólo 6 a la mas arcaica de Aras

${ }^{12}$ De ellas, sólo una remite a la forma propia de Martiño. 
con 50 cada uno ${ }^{13}$, Suero con 42, Gil con 37, Sancho con 36, Luis con 32, Francisco con 28, Men con 27, Lorenzo con 20, Bernal con 18, Gutierre con 16, Jácome y Enrique con 14... Tras estas simples cuantificaciones no es difícil descubrir algunas tendencias destacables en relación con el avance o retroceso de ciertos antropónimos, como los de Juan, Pedro, Álvaro, Fernando o Alfonso, cuya frecuencia aparenta ser todavía muy semejante a la alcanzada en periodos anteriores. No puede decirse lo mismo, por el contrario, respecto a otros, como los de Ruy, Payo, Sueiro, Men, Sancho, García o Gutierre, cuyo paulatino retroceso se aprecia sin dificultad según avanza el periodo contemplado.

En cuanto a la antroponimia femenina la cuantificación de las entradas arroja unos resultados igualmente ilustrativos. Debe advertirse, no obstante, que la presencia de la mujer en la documentación - con independencia de su situación parental como esposa, hermana, madre o hija- es algo menor, en razón naturalmente del papel que en la sociedad medieval en general le correspondía $^{14}$. De ahí, por consiguiente, que el número absoluto de las entradas registradas sea ahora más reducido:

Antropónimo

María

Teresa

Constanza

Inés

Mayor

Leonor

\section{Entradas}

$354^{15}$

$233^{16}$

167

152

152

129

Por debajo del centenar de entradas se sitúan dos antropónimos de todavía cierta solera en aquel periodo: el de Sancha con 86 y el de Elvira con 83. A mayor distancia ya se sitúan los de Isabel y Beatriz con 54, Aldonza con 49, Juana con 46, Berenguela con 41, y en un último grupo se sitúan los de Urraca con 30, Violante y Mencía con 23 cada uno, Aldara con 21, Catalina con 20, Clara con 13, Francisca con 12, Milia con 10... En el caso de los antropónimos femeninos también pueden apreciarse algunas tendencias destacables: el de Inés, por ejemplo, experimenta en el periodo contemplado un indudable incremento, mientras que los de María, Leonor, Teresa, Constanza o Mayor, parecen mantenerse estables. No ocurre esto, en cambio, con los de Sancha, Milia, Berenguela, Aldonza, Urraca, Aldara, Violante o Mencía, cuyo retroceso aparenta ser cada vez más acusado.

\footnotetext{
${ }^{13}$ De las 50 entradas correspondientes al segundo, 9 remiten a la forma propia de Estevo.

${ }^{14}$ Una aproximación al tema en C. PALLARES MÉNDEZ, Las mujeres en la sociedad gallega bajomedieval, en Relaciones de poder, de producción y parentesco en la Edad Media y Moderna, R. Pastor (comp.), Madrid, 1990, págs. 351-373.

${ }^{15}$ De ellas, 17 remiten a la forma propia Mariña y 5 sólo a la de Marina.

${ }^{16}$ De ellas, sólo 2 remiten a formas más arcaicas, como Tarasie o Terasia.
} 


\section{LOS REPERTORIOS ONOMÁSTICOS FAMILIARES \\ DEL ANTROPÓNIMO REGULAR \\ A LA ASOCIACIÓN DEL PATRONÍMICO}

El antropónimo, como es natural, adquirió rápidamente significaciones nuevas, valores añadidos, al ser utilizado para perpetuar el recuerdo de un titular anterior. Esto dio paso muy pronto al uso regular de uno o varios antropónimos dentro de cada estirpe $\mathrm{y}$, al cabo, a la conformación de pequeños repertorios familiares perfectamente estructurados. La evocación de algunos casos resulta muy expresiva del hecho: el de Payo entre los Mariño y el de Ruy entre los Soga, el Vasco y el Gonzalo entre las dos ramas diferenciadas de los Ozores - los de Teanes y los de Orcellón, integrados después en los Ulloa-, el Martín o el Esteban entre los Xunqueiras, el García, el Diego y el Pedro entre los Sarmiento, el Alonso y el García entre los Lanzós, el Vasco y el Ares entre los Parga... Esta nómina de recurrencias antroponímicas, cuyo valor es meramente ilustrativo, puede ampliarse con algunos nombres femeninos de uso igualmente regular en ciertos linajes. Basta recordar, como ejemplo, el caso bien significativo que se produce en el linaje de los Mariñas a partir del matrimonio de Martín Sánchez, artífice de su ascenso a raíz de la guera civil del XIV, con doña Berenguela de Moscoso, hija de Lope Pérez de Moscoso, señor de Altamira, y de doña Berenguela Gómez, su mujer. Y ciertamente, el nombre de aquélla adquirió significación propia en el linaje del marido al repetirse en las tres primeras generaciones - hija, nieta y segunda nieta-, incrementándose sensiblemente esta misma recurrencia en las siguientes, sobre todo durante la primera mitad del siglo $\mathrm{XVI}^{17}$.

No obstante, el renombre o apellido y no desde luego el antropónimo sólo fue el que terminó por identificar al linaje, con lo que el viejo patronímico, que en el momento anterior había servido para expresar la filiación, quedó en muchos casos fosilizado. Son bien conocidos, por su especial resonancia, el Gómez de los Lira y también de los Deza, quienes lo alternaron con el Suárez, igualmente característico en la estirpe, el Páez de los Biedma, el González, el Rodríguez o el Guillélmez de los Bendaña, el Sánchez de los Gres, el López de los Lemos, los Aguiar y los propios Saavedra, el Ares de los Noguerol y el Aras de los Berbetoros, el Núñez de los Isorna, el Fernández de los Andeiro, o el Díaz de los Cadórniga.

Rápidamente, casi inevitablemente, la transmisión de los antropónimos asociados a los patronímicos, en razón de su uso por un antecesor de mayor o menor relieve social -o por su sola importancia en la historia familiar-, se convirtió en un rasgo propio, singular, entre los linajes nobles

${ }^{17}$ Este Martín Sánchez das Mariñas era hijo de Gómez Pérez das Mariñas, señor del coto de Cecebre, y de doña Teresa Alfonso, su mujer. Una cumplida información sobre el linaje, aunque hoy revisable, en C. VAAMONDE LORES, Gómez Pérez das Mariñas y sus descendientes, La Coruña, 1917 . 
del último periodo del medievo. Con esta nueva costumbre, ciertamente, aquéllos procuraron preservar la memoria - la acordança- de los personajes más tempranos o sobresalientes, cuyo prestigio social se deseaba trasladar a los nuevos vástagos así bautizados, lo que repercutía directamente también en la mayor cohesión interna del grupo familiar. Este y no otro es el sentido de las más tempranas asociaciones, como la Juan Fernández en el linaje de los Limia, rama menor ya mencionada de los Traba. Su origen estuvo en uno de los más tempranos y destacados personajes de la estirpe, cuya personalidad ya quedó anotada como titular de la tenencia de Limia, así como mayordomo mayor y alférez mayor en tiempos de Alfonso IX ${ }^{18}$, portándola sucesivamente un nieto suyo, que fue pertiguero mayor de Santiago en tiempos de Alfonso $\mathrm{X}$ y adelantado mayor de Andalucía ya en los de Sancho $\mathrm{IV}^{19}$, un segundo nieto, que fue arzobispo de Santiago entre los años 1330 y $1338^{20}$, y finalmente un tercer nieto, que falleció a fines de 1365 o comienzos de $1366^{21}$.

Esta y otras muchas asociaciones, que adquirieron significación y notoria reputación en la Galicia de la segunda mitad del XIII, fueron motivo de inspiración o de clara imitación entre los linajes más modernos o de menor relieve y fortuna. De ahí, pues, el Munio Fernández de los Rodeiro, el Fernán Pérez de los Andrade ${ }^{22}$, el Gil Fernández y el Fernando Gil de los primeros Taboada $^{23}$, así como el Ares Pérez y el Lope Pérez de sus sucesores, el Vasco Pérez de los Quiroga, o el Martín Rodríguez de los Xunqueiras. Esta última, por ejemplo, también tuvo su origen en el primer personaje de relieve en el linaje, que se documenta como juez de Postmarcos en los últimos años del

${ }^{18}$ J. GonZÁLEZ, Alfonso IX, vol. I, pp. 227, 323 y 324.

${ }^{19}$ M. GAIBRoIS, Sancho IV, vol. I, p. 13.

${ }^{20}$ A. LÓPEZ FerreIro, Historia de la Iglesia de Santiago, vol. VI, pp. 87-88.

${ }^{21}$ La muerte de este último Juan Fernández se consigna documentalmente en M. B. VAQUERO DíaZ, Colección diplomática do mosteiro de San Salvador de Celanova (S. XIII-ẌV), Santiago, 2004, vol. I doc. 154, pp. 211-212. Un panorama del linaje, aunque sin algunas de las precisiones ahora incluidas, en E. PARDO DE GUEVARA Y VALDES, Parentesco y nepotismo", nepotismo. Los arzobispos de Santiago y sus vinculos familiares en los siglos XIV y XV en R'. Izquierdo Perrín (coord.), Los coros de las catedrales y monasterios: arte y liturgia, Fundación Pedro Barrié de la Maza, A Coruña, 2001, pp. 67-70.

${ }^{22} \mathrm{La}$ estirpe de los Andrade presumió ya desde muy temprano de tener su origen en los Traba. La pretension, hoy mucho más que dudosa, fue reiteradamente divulgada por los genealogistas gallegeos. De cualquier forma, el primer gran referente en el linaje fue Fernán Pérez $O$ Boo, que vivió en la segunda mitad del XIV, lo que explica el mantenimiento de la recurrente asociación de su nombre y patronímico, que hasta mediados del XVI portaron cuanto menos hasta siete personajes diferentes, si bien sólo dos de ellos fueron realmente herederos de la Casa, que había sido conformado y encumbrado por el primero. El linaje ha suscitado diversos estudios monográficos en el último cuarto del siglo pasado, aunque con muy variada fortuna y acierto. Véanse, sólo como ejemplo, la memoria de licenciatura inédita de M.C. LORENZO FOLGUEIRO, El linaje de la Casa de Andrade, Madrid, 1974, así como las dos síntesis publicadas: V. H. ZOLTAN, Los Andrade. Una bibliografía histórica " "Anuario Brigantino" 14 (1994), pp 167-185, y J. GARCíA ORO, Don Fernando de Andrade conde de Villalba, Xunta de Galicia, 1993. Una clarificación genealógica más reciente y bien documentada, aunque sólo se refiere a las primeras generaciones de la estirpe y todavía sin precisar su nexo de unión con las más recientes y conocidas del linaje de Fernă "Pérez $o$, Boo, en C. CCASTRO ALVAREZ y J.L. LóPEZ SANGIL, La genealogía de los Andrade, "Cátedra", 6 (1999), págs. 189-216.

${ }^{23}$ Las frecuentes homonimias entre los Limia y los Taboada inducen a considerar la posible derivación genealógica de estos, lo que sin embargo no ha sido posible documentar todavía. G. LEDO, Palos, fajas y jaqueles, Lugo, 1997, págs. 77-80. 
$\mathrm{XIII}^{24}$, y se repitió en su nieto, documentado también como juez de Postmarcos poco después de mediar el siglo $\mathrm{XIV}^{25}$, en su segundo nieto y todavía, asimismo, en su tercer nieto, documentado como alcalde de Santiago justo antes de mediar el siglo $\mathrm{XV}^{26}$.

La generalización de esta misma costumbre se aprecia incluso una vez agotada la línea de varón, al sobrevivir a través de la línea femenina por la que se transmiten los patrimonios. Esto se documenta, por ejemplo, entre los descendientes de un personaje de la pequeña nobleza de mediados del XIV, Ares Conde, cabeza del linaje de los Monterroso y señor de la Casa de San Miguel das Penas ${ }^{27}$, cuya línea de varonía se agotó en su nieto homónimo, aunque aquel antropónimo y alcume se repitieron con insistencia entre los descendientes de la hija y heredera de este último, doña Leonor López de Aguiar, mujer del primer Fernán Ares Noguerol, en cuya descendencia continuó la Casa de San Miguel das Penas ${ }^{28}$. De esta forma, en los últimos años del siglo XVI, todavía puede documentarse un personaje homónimo, séptimo nieto del primero, en quien recayó la sucesión de aquel viejo solar ${ }^{29}$.

Casi de inmediato, la documentación empieza a reflejar con abundancia la generalización de la costumbre, de manera que no resulta extraño identificar dentro de un mismo linaje a varios personajes homóminos coetáneos. Entre el grupo de los Valcárcel y Balboa se documenta el caso del García Rodríguez, singularísimo a mi parecer, pues su desconocimiento ha dado pie a más de un desatino histórico que todavía goza de crédito; en realidad, esta combinación onomástica fue llevada por no menos de seis personajes diferentes, entre ellos figura el más famoso de todos, que fue adelantado mayor de Galicia al poco de concluir el primer cuarto del siglo $\mathrm{XIV}^{30}$. Así, en efecto, el padre y el abuelo del propio Adelantado, a los cuales se documentan muy bien en el último tercio del siglo XIII y los primeros años del XIV. Además de estos, se llamaron también así un hijo del Adelantado,

\footnotetext{
${ }^{24}$ ADM, Junqueiras, leg. 1, núms. 81 y 82, leg. 3, núms. 99 y 109, y leg. 4, núm. 42.

${ }^{25}$ Ibidem, leg. 1, núm. 55, y leg. 3, núm. 111 y $138 .$.

${ }^{26}$ Este personaje casó con doña Inés Gómez de Sotomayor, hija del famoso Payo Gómez de Sotomayor. En ella hubo a Esteban Rodríguez de Xunqueiras, que fue uno de los más destacados representantes de la estirpe. Información sobre la sucesión genealógica en E. PARDO DE GUEVARA Y VALDÉS, Parentesco y nepotismo, p. 87, nota 99.

${ }^{27}$ Este personaje era fallecido ya en 26 de julio de 1383, fecha en que otorgó testamento su viuda, dona Teresa García. AHN, Clero, carp. 1262, núm. 8.

${ }^{28} \mathrm{El}$ segundo Ares Conde de Monterroso, nieto del primẹro, otorgó su testamento en 8 de octubre de 1439, mientras que su hija doña Leonor lo hizo, siendo viuda y muy anciana, en 30 de junio de 1502. ADM, San Miguel das Penas, leg. 1, núm. 84.

${ }^{29}$ Este séptimo nieto fue Ares Conde de Taboada -el sexto en la sucesión de homónimos del linaje-, al cual se documenta como señor de la Casa de San Miguel das Penas a finales del XVI y comienzos del XVII. Así, por ejemplo, en la escritura de mejora otorgada a favor de Pedro de Gayoso, primogénito de Fernán Ares Noguerol, su primo segundo, en 30 de agosto de 1590. Ibidem, leg. 1, núm. 90.

${ }^{30}$ Importa precisar que el ejercicio de este oficio se documenta en 1328 , en coincidencia con la caída del todopoderoso privado don Alvar Núñez Osorio, motivada por el motín de Valladolid de aquel mismo año. Los hilos de la maniobra fueron movidos por don Juan Manuel y por frey Fernan Rodríguez de Balboa, prior de San Juan y pariente de este García Rodríguez. Véase, S. DE MOXÓ, La sociedad política castellana en la época de Alfonso XI, "Cuadernos de Historia", 6 (1975), pp. 255-257.
} 
que fue como comendador de Castrotorafe en la Orden de Santiago, y un nieto - hijo de doña Constanza de Valcárcel y de Juan Freire de Andrade-, que fue el que recibió del conde don Pedro las Puentes de Eume, hoy Puentes de García Rodríguez, allá por 1372. De este último personaje, fallecido poco después de 1407 y casado con doña Inés Fernández [de Moscoso], quedó entre otros hijos una doña Leonor García de Valcárcel, la cual casó a su vez con Lope Alfonso de Lemos, señor de Ferreira, y de ellos fue nieto un nuevo García Rodríguez de Valcárcel, que se documenta al poco de mediar el cuatrocientos como capitán de las gentes de guerra del conde de Lemos ${ }^{31}$.

Desde mediados de la centuria anterior fueron ya de uso regular, por ejemplo, el Fernán Yáñez o el Juan Fernández entre los Sotomayor, el Vasco López o el Lope Sánchez entre los Ulloa, el García Díaz entre los Mesía, el Lope Alfonso o el Alonso López entre los Lemos, el Vasco Gómez entre los Seixas, el Lope Pérez entre los Moscoso, el Ares Vázquez o el Vasco Pérez. entre los Vaamonde, el Lope Núñez entre los Montenegro, el García Díaz o el Pedro Díaz entre los Cadórniga, el Alonso López entre los Aguiar y Saavedra, o el Diego Sánchez o el Fernán Díaz entre los Ribadeneira, que en sus primeras cinco generaciones fueron llevados respectivamente por cinco y siete personajes diferentes ${ }^{32}$. Estas mismas asociaciones de antropónimo y patronímico se dieron también entre las mujeres, como la de Leonor García, que se repitió en el grupo de los Valcárcel, la de Teresa Rodríguez en el de los Aguiar, la de Milia Vázquez en el de los Quiroga, la de Teresa Fernández en los Ribadeneira, o las de Leonor Rodríguez o Sancha Fernández entre los Parga.

El sentido o significado de estas asociaciones, cuya recurrencia no se vincula sólo a las líneas directas ni disminuye en ciertas líneas femeninas, se aprecia mucho mejor cuando se contextualizan dentro de una misma generación, tratando de explicar la razón de los nombres y patronímicos llevados por cada uno de los vástagos de un mismo matrimonio. Así por ejemplo, entre los hijos de García Rodríguez el Adelantado y de doña Teresa Yáñez Sarraza, su mujer ${ }^{33}$, se descubren tres casos explicables; en primer

\footnotetext{
${ }^{31}$ Véase, M. MARTínez MARTíneZ, Cartulario de Santa María de Carracedo, Instituto de Estudios Bercianos, vol. I, docs. 528, 553 y 584, pp. 381, 395 y 416, y vol. II, docs. 688,729 y 884, págs. 52, 76-77 y 189-190, y E. CAL PARDO, Catálogo de los documentos medievales, escritos en pergamino, del Archivo de la Catedral de Mondoñedo, Lugo, 1990, doc. 1329, pág. 504. Un panorama actualizado sobre las filiaciones y parentescos del linaje de los Valcárcel ẹn E. PARDO DE GUEVARA Y VALDÉS, El linaje de la Casa de Tor. Anotaciones críticas”, estudio e introducción al libro de Benjamín de Prado, en El linaje de la Casa de Tor, Diputación Provincial de Lugo, Lugo, 2007.

${ }^{32}$ El recuento se hace a partir del matrimonio de Fernán Díaz de Ribadeneira y doña Berenguela López, documentado en las últimas décadas del XIV, que puede ser considerado como el punto de partida de este linaje nuevo.

${ }^{33}$ Esta doña Teresa fue hija de Juan Vázquez Sarraça y de su primera mujer, doña Teresa Alfonso de Deza. Esta última, a su vẹz, lo fue de Alfonso Gómez de Deza, o dẹ Provaos, y de doña María Fernández Vịcos, su mujer, de los cuales también quedaron por hijos doña María Vicos, doña Aldonza, mujer de Pedro Suárez Gallinato, y el famoso Alfonso Suárez de Deza, que llamaron el Churruchao. Véase, Libros velhos de linhagens, vol. I, p. 173. Para J. A. de Sotto Maior Pizarro, șin embargo, ésta doña Teresa Yáñez Sarraça se situaría en una generación anterior, como hija de Vasco Pérez Sarraça y, por tanto, hermana del Juan Vázquez y concuñada de los mencionados, lo que no parece muy probable, más aún cuando no se apunta así en el Livro
} 
término, el primogénito y heredero, que como ya se ha dicho llevó el mismo antropónimo y patronímico de su padre y abuelo, después a Alonso Suárez llamado así en recuerdo del hermano de su abuela materna, el famoso Alonso Suárez de Deza el Churruchao ${ }^{34}$, y por último a doña María Vicos, en recuerdo a su vez de otra hermana de su abuela materna, doña María Fernández Bicos ${ }^{35}$. Nada preciso puede decirse, en cambio, respecto a los otros hijos -Álvar Rodríguez, Constanza García y doña Leonor García-, aunque sus nombres y patronímicos se repiten entre la parentela de los propios Valcárcel. Estas mismas transmisiones se aprecian en la generación siguiente; así, entre los hijos de la mencionada doña Constanza García y Juan Freire de Andrade, se reconoce al primogénito, García Rodríguez, llamado así en recuerdo de su tío o mejor de su abuelo materno, el famoso Adelantado, y a Alonso Suárez de Lanzós, cuyo nombre y patronímico recordaban a su vez al mencionado tío, hermano de la madre. El valor o influjo de este parentesco transversal en el contexto de las costumbres onomásticas pone de relieve la importancia del prohijamiento, entendido como una extensión o prolongación de ese vinculo parental que articula la transmisión de patrimonios entre parientes homónimos; un buen ejemplo, entre muchos, lo ofrece un destacado personaje del petrismo gallego, don Pedro Fernández de Bolaño, quien al no alcanzar descendencia legítima solicitó - y en 1389 la obtuvo- la pertinente autorización regia para vincular sus bienes en un sobrino suyo del mismo nombre, hijo de Alonso López de Saavedra y de doña Sancha Núñez, su mujer ${ }^{36}$.

\section{LA AFIRMACIÓN DEL LINAJE \\ EL RENOMBRE Y LA TRANSMISIÓN DE LOS ALCUMES}

Junto a estas pautas onomásticas, concretadas en la comentada asociación de antropónimos y patronímicos, se advierte también el progresivo

Antigo de Linhagens. Véase, J.A. DE SOTTO MAYOR PIZARRO, Linhagens medievais portuguesas. Genealogías e estratégias, Porto, 1999 , vol. II, p. 191. También, Fundación y dotación del monasterio de Carracedo, según el manuscrito de Fr. Jerónimo de Llamas, edición de F. González González, Zaragoza, 1993, p. 137.

${ }^{34}$ Este Alonso Suárez de Deza se llamó así, por su parte, en recuerdo de su segundo abuelo, Alonso Suárez Sarraza. Este personaje, hijo de Suero Ares de Valladares y hermano de Pedro Suárez Sarraza, casó con dona Teresa Eanes de Deza, dejando en ella a Fernando Alfonso, muerto sin sucesión, y a doña Mayor Alfonso de Deza, mujer de Gómez Enriquez de Probaos y madre del Alfonso Gómez de Deza, o de Probaos. Véase, PORTUGALIAE MONUMENTA HISTORICA, Livros velhos de linhagens, edición crítica de J. Mattoso, vol. I, Lisboa, 1980, vol. I, p. 173.

${ }^{35}$ Esta María Vicos, hija del Adelantado y viuda de Payo Varela, fue la tercera mujer de Rui Freire de Andrạ́de, padre del famoso Fernán Pérez de Andrade $o$ Boo. Este matrimonio guarda indudable relación con el que contrajo su hermana doña Constanza con Juan Freire de Andrade, hermano del Rui Freire. De estos ûltimos quedaron varios hijos, entre ellos un nuevo Alonso Suárez, que llevó el renombre de los Lanzós. AHN, Clero, Carp. 492, núm. 7.

${ }^{36}$ Este otro Pedro Fernández de Bolaño, tercero en su estirpe, se documenta en el primer tercio del XV como merino mayor del duque de Arjona. Su sepulcro con yacente, contiguo al de su hermano Rodrigo Alonso de Saavedra -o Bolaño-, se conserva todavia en la antigua conventual franciscana de Lugo. ADM, Junqueiras, leg. 1, núm. 97. 
avance de los renombres o apellidos propiamente, cuya aceptación - acostumbró a imponerse desde fuera - y posterior transmisión comienza a generalizarse ya al mediar el siglo XIII, alcanzando a singularizar la estabilidad de los linajes al avanzar la centuria siguiente. Este nuevo paso resulta decisivo, por consiguiente, en el largo proceso de cristalización de aquéllos, pues con el uso del renombre los miembros de las distintas ramas derivadas de un antepasado común tomaron conciencia plena de pertenecer a un mismo linaje.

Como ya se ha apuntado más atrás, en la conformación de los renombres fue mayoritario el protagonismo de los topónimos, que acostumbraron a aludir al solar o señorío de origen, cuando no a una función o privilegio determinado: el gobierno de una importante tenencia, por ejemplo, explica el nombre de familia portado por los Limia, rama menor de los Traba, mientras que la posesión de un simple castillo o fortaleza lo explicaría en el caso de los Andrade. Y junto a este tipo de renombres de referencia exactamente geográfica, que fueron en efecto la gran mayoría, hubo otros de alusión más precisa, como los que se hacían eco de la fisonomía urbana y precisaban el lugar o rincón exacto donde el linaje tenía su solar o asiento; ocurrió así, por ejemplo, con los Ocampo - o do Campo - o con los Vidal do Camiño, ambos compostelanos. Pese a todo, hubo casos también en que la sola singularidad del antropónimo portado por un primer personaje referencia hizo innecesario que el patronímico derivado se concretara o complementara, según la nueva costumbre, con un segundo cognomen o renombre. De esta forma, la filiación expresada por aquél no se fosilizó exactamente, sino que mantuvo su valor como señal de identidad, perpetuándose conscientemente después en las sucesivas generaciones: el caso, que es paralelo - pero no coetáneo- a los Osorio y los Ponce leoneses, o a los más alejados Manrique, se ilustra muy bien aquí con el linaje de los Ozores, forjado en torno a la torre de Teanes, cerca de la villa de Salvatierra, a partir de Osorio Eáns Gago ${ }^{37}$.

Mención aparte merecen otro tipo de renombres o apellidos, pues a través de ellos se evidencia todavía mejor su valor en la afirmación social del linaje y en la perpetuación de su propia memoria genealógica. Me refiero ahora, a la transmisión de los apodos o alcumes y su posterior aceptación como renombre propio del linaje. Pese a su singularidad - extraordinaria en Castilla y muy frecuente en Portugal-, no fue una fórmula ciertamente extraña en Galicia. Aquí se documenta, por ejemplo, en los Maldonado, cuyo renombre procedió del alcume aplicado a uno de sus más tempranos personajes, Nuño Perez o Maldonado; también en los ya anotados Sarraza, cuyo renombre se originó a su vez en el alcume de Pedro Suárez de Vallada-

\footnotetext{
${ }^{37}$ A fines del XIII se documenta al personaje que cabe situar a la cabeza del linaje, Osorio Eans Gago, quien alcanzó un cierto relieve con intereses en ambos lados de la raya de Portugal, así como a varios hermanos suyos, nietos de un don Juan Núñez Gago. El hijo de este personaje, Vasco Ozores, consolida la posición del linaje -el renombre se fija entonces- en torno a la villa de Salvatierra. Véase, E.PARDO DE GUEVARA Y VALDÉS, Osorio Eans Gago y su descendencia. Noticia de un linaje galaico-miñoto de comienzos del siglo XIV, en Estudos em homenagem ao Professor Doutor José Marques, Universidade do Porto, 2006, vol. I, pp. 479-497.
} 
res, al que llamaron $o$ Sarraça, a quien puede situarse en la primer mitad del $\mathrm{XIII}^{38}$, o asimismo en los Pardo de Cela, salidos del entorno de Betanzos y cuyo renombre tomaron, a su vez, de otro personaje también temprano, don Fernando Pérez, dictus Pardo, igualmente de la primer mitad del XIII ${ }^{39}$.

Menos conocida es, en cambio, la transmisión o repetición del apodo o alcume como tal; es decir, sin que se aceptara como nombre de familia. Un caso singular y muy ilustrativo, aunque no fue probablemente el primero, lo ofrece un personaje gallego muy conocido en el concierto de la nobleza gallega, famoso además por su protagonismo en la historia política de la segunda mitad del cuatrocientos: don Pedro Álvarez de Sotomayor, conde de Camiña y señor de la Casa de Sotomayor, conocido popularmente como Pedro Madruga, pues - lo recuerda incluso el propio Aponte- madrugaba mucho quando haçía sus cabalgadas. Sorprende descubrir, sin embargo, que el celebrado alcume ya había sido utilizado con anterioridad por otro personaje, miembro de una rama menor andaluza del viejo tronco gallego de los Sotomayor; me refiero al no menos conocido García Méndez de Sotomayor, señor de El Carpio, quien en los primeros años del reinado de Juan II se había distinguido en las guerras con la morisma, particularmente en el cerco de Teba, donde se hizo famoso como Garcí Madruga, dicen que por la malas madrugadas que daba a los moros ${ }^{40}$.

El caso mencionado no es el único, ni siquiera el más ilustrativo de los que pueden documentarse en relación con la pervivencia de los alcumes. Basta recordar, como ejemplo, lo que sucedió en la Casa de Lemos, señoreada en el siglo XIV por dos personajes de excepción: don Pedro Fernández de Castro, al cual llamaron el de la Guerra, que fue uno de los personajes centrales del reinado de Alfonso XI, y su hijo don Fernando Ruiz de Castro, al que llamaron a su vez toda la lealtad de España, resumiendo así su inquebrantable lealtad a Pedro I, cuya legitimidad defendió aún después de los hechos de Montiel. El extraordinario prestigio de estos dos personajes gravitó

\footnotetext{
${ }^{38}$ El personaje era hijo de Suero Arias de Valladares, que figuró en la corte de Alfonso Enríquez, y de su segunda mujer, doña María Alfonso, una de las bastardas de Alfonso IX y de Teresa Gil de Soberosa. Véase, Livros velhos de linhagens, vol. I, Tit. XIII, págs, 173., y J.A. DE SOTTO MAYOR PIZARRO, Linhagens medievais portuguesas, vol. II, pp. 190-194.

${ }^{39}$ Este Fernandus Petri, dictus Pardus se documenta en 1246 al confirmar -junto con su cuñado don Nuño y sus sobrinos don Lope Núñez y doña Mayor Yáñez- una donación de su padre, dominus Petrus Garcie, al monasterio de Monfero, a cambio de lo cual obuvo licencia para enterrarse dentro de su iglesia. Su hijo, llamado ya Pedro Fernández Pardo, se documenta en la segunda mitad del XIII haciendo tuertos al referido monasterio. ARG, Colección de Pergaminos, num. 107.

${ }^{40}$ Este Garcíméndez de Sotomayor, sexto señor de El Carpio y segundo señor de Morente, veinticuatro de Córdoba, fue hijo de Luis Méndez de Sotomayor y de doña Catalina Sánchez Mạnuel de Villodre. Casó con doña María de Figueroaa, hija del maestre don Lorenzo Suárez, dejando en ella abundante descendencia. Al personaje se le documenta entre 1390 y 1440 , particularmente en relación con su presencia en la frontera granadina. Noticias y referencias en M. CABRERA, El señorío del Carpio en el siglo XV, en Aragon en la Edad Media. Siglos XIV-XV, en Homenaje a la profesora Carmen Orcástegui, Zaragoza, 1999, vol. I, pág. 228. Véase, además, Adiciones a la Crónica de Enrique III, BAE vol. 68, Cap. III, pp. 249-250, y Crónica de Juan II, BAE, vol. 68, Caps. XXX y XXXI, pp. 289-290; también, G. ARGOTE DE MOLINA, Nobleza de Andalucía, Jaên, 1866, Caps. IV, pp. 271-275, y CXXVIII, pp. 500-505, y A. LÓPEZ DE HARO, Nobiliario, vol. I, fol. 48, así como una breve mención en Fr. F. DE LA GÁNDARA, Armas y triunfos, hechos heróicos de los hijos de Galicia, Madrid, 1662 (edic. facsimile de Compostela, 1970), fol. 373.
} 
sobre todos sus sucesores en la titularidad de la Casa de Lemos, cuatro de los cuales - del cuarto al séptimo conde - se llamaron exactamente así casi doscientos años después, adornándose incluso con los mismos famosos alcumes; el hecho consta expresamente con el quinto titular, don Pedro Fernández de Castro, que en el acto de su cruzamiento en la Orden de Calatrava, en 17 de abril de 1541, recibió el sobrenombre que había llevado su antecesor homónimo ${ }^{41}$.

En el contexto de estas y otras costumbres onomásticas de uso cotidiano y generalizado se advierte, también por estos siglos, la ocasional feminización de ciertos "apellidos", que no eran utilizados propiamente como renombre, sino como nombre de familia y todavía con parte de su carga semántica intacta. Este fue el caso, por ejemplo, de Mayor García Parda, hija de García Pardo y hermana de Fernán Pardo, de Betanzos, la cual se documenta como monja en Santa Clara de Santiago entre los años 1366 y $1406^{42}$, o también el de doña Leonor Suárez Gallinata, mujer de Ruy López de Montenegro, hermano del primer Tristán de Montenegro que se mencionará más adelante, o el de otras muchas que prefirieron nombrarse en femenino: Parraguesa por Parragués, Arauja por Araujo, Falcona por Falcón, Piñeira por Piñeiro, Salgada por Salgado, Gaudiosa o Gayosa por Gaudioso o Gayoso, Vizosa por Vizoso... Pero esta costumbre no era propia sólo de los grupos de la nobleza media gallega, sino que se extendía a las capas populares y también cuando se tomaba al renombre del marido; así el caso de una María Telleira, mujer de Juan Telleiro, a los cuales se documentan en 1482 con motivo de un pleito del monasterio de Carboeiro sobre la granja de Mesego, donde aquellos habían levantado una casa ${ }^{43}$. Pese a que los ejemplos mencionados remiten en general al espacio y cronología propuestos, Galicia al fin de la Edad Media, lo cierto es que la costumbre antecedente tuvo vigencia en un ámbito espacial y temporal mucho más amplio. Salazar y Acha lo apunta muy bien al retrasarla a los tiempos del Siglo de Oro, rastreando su huella hasta la misma actualidad, aunque precisa ya su aire típicamente popular y su mayor arraigo cuando se trata de renombres originados en apodos o alcumes $^{44}$.

${ }^{41} \mathrm{~F}$. FERNÁNDEZ DE BETHENCOURT, Historia genealógica y heráldica de la Monarquía española , vol. IV, Madrid, 1897 , pág. 536. Información cumplida sobre estos cuatro personajes en E. PARDO DE 'GUEVARA Y VÁLDS, Don Pedro Fernández de Castro, VII conde de Lemos (1576-1622), Santiago de Compostela, 1997, 2 vols.

${ }^{42}$ C. RoDRíGUEZ NÚÑEZ, El papel de la mujer en la sociedad medieval, Lugo, 1993, pág. 282.

${ }^{43} \mathrm{AHN}$, Clero, carp. 1554-20.

${ }^{44}$ Véase, J. DE SALAZAR Y ACHA, Génesis y evolución histórica del apellido, págs. 30-31. 


\section{UNA HUELLA CULTURAL EL INFLUJO DE LA MATERIA DE BRETAÑA EN GALICIA}

Aunque es una cuestión relacionada sólo indirectamente con los lazos de parentesco y la identidad, no está de más apuntar ahora que las costumbres antroponímicas evidencian las más de las veces, como es natural, trasfondos ideológicos y culturales de muy diverso signo. Un testimonio muy ilustrativo es la aceptación y uso de nombres procedentes de la literatura artúrica y, en general, de todo el ciclo bretón, moda que tuvo una aceptación irregular en los distintos territorios de la corona de Castilla, como ha sido puesto de relieve hace algunos años ${ }^{45}$.

Pero Galicia, en este contexto, ofrece un panorama muy interesante por el número y variedad de los testimonios que se pueden documentar. Las razones que podrían explicar el mayor influjo aquí de esta moda onomástica son muy diversas; entre ellas estarían, naturalmente, las relaciones con el vecino reino portugués, sobre todo a partir de la debacle del petrismo y de las consiguientes pretensiones inglesas, o la fluidez misma de los movimientos y relaciones comerciales de los puertos atlánticos gallegos, reconocida ya desde mucho atrás ${ }^{46}$. No obstante, es difícil adivinar hasta qué punto hubo en Galicia un conocimiento especialmente intenso y directo de los ciclos literarios que inspiraron aquella moda antroponímica. En realidad, por lo que se sabe, la circulación de estas obras por el occidente peninsular - particularmente por Galicia- no parece que trascendiera a las esferas más elevadas de la nobleza territorial, pese a la receptividad cultural que también se habría articulado a través del Camino de Santiago y al eco mismo de aquellos ciclos en algunas obras portuguesas o castellanas, como el Livro de Linhagens do conde don Pedro y la Crónica de 1404 o las Generaciones y semblanzas de Pérez de Guzmán y las Bienandanzas e fortunas de García de Salazar, estas dos últimas escritas al poco de mediar el siglo $\mathrm{XV}^{47}$. Pese a todo, es indudable que

\footnotetext{
${ }^{45}$ Véase, I. BECEIRO PITA, Modas estéticas y relaciones exteriores. La difusión de los mitos artúricos en la corona de Castilla (S. XIII-comienzos del XVI), "En la España medieval", 16 (1993), pp. 135-167.

${ }^{46}$ Así, por ejemplo, los puertos gallegos de La Coruña, Ribadeo, Vivero, Betanzos, Ortigueira, Cedeira, Ferrol, Bayona, La Guardia, Pontevedra, Padrón y Noya, figuraban entre los más importantes que estaban autorizados para el tráfico de mercancías poco después de mediar el siglo XIII. Véase, J.I. RUIZ DE LA PEÑA, El nacimiento de las villas costeras cantabro-atlánticas y su instrumentación jurídica (siglos XII-XIII), en El fuero de Laredo en el VIII centenario de su concesión, Santander, 2001 , p. 164.

${ }^{47}$ Un panorama reciente sobre la difusión de la literatura artúrica y, en general, de toda la materia de Bretaña en Galicia en S. GUTIÉRREZ GARCíA y P. LORENZO GRADÍN, A literatura artúrica en Galicia e Portugal na Idade Media, Santiago de Compostela, 2001. Véase, además, M. PASTOUREAU, Lo'enromencement du nom. Etude sur la diffusion des noms des héros arthuriens à la fin du Moyen Age, en Couleurs, images, symboles, París, 1990, págs. 112-125, y sobre las bibliotecas bajomedievales, cuyo estudiọ alumbra la cuestión tratada, véase como panorama general, aunque con ilustrativas informacipones, CH. B. FAULHABER, Libros y bibliotecas en la España medieval, Valencia, 1987, e I. BECEIRO PITA. Bibliotecas y humanismo en el reino de Castilla. Un estado de la cuestión, "Hispania", L/2 (1990), pp. 827-839, quien dos años después trató con mayor detenimiento la difusión de los mitos artúricos en La fascinación pour la "matière de Bretagne" dans la noblesse castillane du Moyen Age tardif, en 1491. La Bretagne, terre d'Europe, Brest-Quimper, 1992, pp. 325-337.
} 
aquellos héroes literarios circularon como modelos caballerescos en el imaginario medieval, particularmente en las pequeñas pero brillantes cortes señoriales, como aquella de don Rodrigo Gómez, el último de los Traba, que se hizo famosa en la Galicia de la primera mitad del XIII ${ }^{48}$, o la del duque don Fadrique de Arjona, que ya en las primeras décadas del XV sería el lugar de encuentro de los más celebrados poetas y trovadores del momento; lo recordó López Ferreiro - siguiendo al marqués de Santillana- que a don Fadrique plogole mucho la sciencia de trovar y gustó de tener en su casa grandes trovadores, especialmente Fernán Rodríguez Puertocarrero, Juan de Gayoso y Alfonso de Morana [Moraña?], a los cuales el propio historiador compostelano añadía a Juan Rodríguez de Padrón, que allí pudo encontrarse con el famoso Macías ${ }^{49}$.

Como ampliación a lo ya sabido, no está de más apuntar ordenadamente ahora algunos casos bien conocidos en el concierto de la nobleza gallega, anotando siempre que sea posible su origen, momento y posición social. En primer término, cabe mencionar a las distintas mujeres que recibieron el nombre de la reina doña Ginebra. Así, al margen de una primera doña Ginebra de Ribeiro, mujer de un Nuño Freire de Andrade, pero de muy probable naturaleza portuguesa ${ }^{50}$, las más tempranas que pueden documentarse en Galicia son doña Ginebra das Mariñas, cuya trayectoria vital se sitúa en el tercer tercio del siglo $\mathrm{XV}^{51}$, y su sobrina doña Ginebra de Haro, que traspasaría ya los umbrales del quinientos ${ }^{52}$. De estos mismos años son asimismo las otras dos ginebras que se documentan dentro del linaje

\footnotetext{
${ }^{48}$ Este don Rodrigo Gómez no alcanzó descendencia de su matrimonio con doña Mayor Alonso de Alburquerque, hija de Alonso Téllez de Meneses, el de Alburquerque, y no de Âlfonso IX como algunos repiten todavía. Respecto a su corte señorial, se sabe que en la misma confluyeron una seriẹ de trovadores -Nuño Fernández de Mirapeixe entre ellos- cuyas composiciones estaban influenciadas por la materia de Bretaña y, particularmente, por el ciclo artúrico; véase, Y. FRATESCHI VIEIRA, En cas dona Maior: os trovadores e a corte senhorial galega no século XIII,
Noya, 1999.

${ }^{49}$ Véase, Historia de la Iglesia de Santiago, vol. VII, Santiago,1904, pp. 44 y 147-178. No obstante, recientes investigaciones precisan la cronología de este Macías en un tiempo bastante anterior. Véase M. ROMANÍ y P.S. OTERO PINEYRO-MASEDA, Macías, un juglar del siglo XIII, "El Museo de Pontevedra", 59 (2005), pp. 29-38.

${ }^{50}$ Esta doña Ginebra do Ribeiro estuvo casada efectivamente con un Nuño Freire de Andrade, que fue el primero de los cuatro hijos que una Clara Martíz hubo en el famoso Nuño Freire de Andrade, maestre de la Orden de Cristọ. La descendencia de esta doña Gịnebra, avecindada en Portugal, así como la grafía de su propio renombre, permiten aventurar sin muchas reservas su naturaleza portuguesa. Véase, INSTITUTO DE ESTUDIOS GALLEGOS "PADRE SARMIENTO", Archivo Genealógico de Eduardo Pardo (en adelante sólo AGEP) Andrade, núm. 3.

${ }^{51}$ Hija de Gómez Pérez das Mariñas y de doña Teresa de Haro y Acuña, su mujer, que lo era a su vez de doña Ginebra de Acuña -hija del exiliado portugués Martín Vázquez de Acuna, conde de Valencia de don Juan-. La doña Ginebra citada en el texto casó con Luis de Acevedo y con Ruy Yáñez de Sotomayor. ADM, Cillobre, leg. 1, núm. 6.

${ }^{52}$ Hija de Fernán Pérez Parragués, regidor de La Coruña, y de su segunda mujer, doña Constanza das Mariñas, que era hermana de la ya citada doña Ginebra. Esta otra doña Ginebra fue dotada por sus padres en 7 de agosto de 1508, casando de seguido con Suero Gómez de Sotomayor, señor de Sobrán, en el que dejó sucesión. AGEP, Parga, núm. 18; además, V. DE APONTE, Recuento, pp. 124-125.
} 
Noguerol, la una hija de Fernán Ares $^{53}$ y la otra de Juan de Noguerol ${ }^{54}$. La nómina puede ampliarse todavía con otras tres identificaciones: una Ginebra de Toro, que en los primeros años del XVI se documenta como sobrina de Sancho Sánchez de Ulloa e hija, acaso, del arzobispo Fonseca ${ }^{55}$, así como una Ginebra de Deza, cuya origen no puede precisarse, aunque consta que por el año 1514 estaba casada con un mercader orensano, Gómez Moure, originario probablemente de la villa de Chantada, al sur de la provincia de Lugo ${ }^{56}$, y finalmente una doña Ginebra de Araujo, que en la primera mitad del XVI figura casada con Vasco de Romay, señor del coto de Quadro ${ }^{57}$.

No son extraños, tampoco, los testimonios que ponen de relieve el influjo del celebrado sir Lançelot, como lo prueba el Lanzarote Mariño, que se documenta en las últimas décadas del $\mathrm{XV}^{58}$, o la sucesión de personajes homónimos que terminó por dar una impronta singular a la estirpe cedeiresa de los Lago ${ }^{59}$. También tuvo cierta aceptación el nombre de Lionel, uno de los parientes partidarios de sir Lançelot. Así, el Lionel de Montaos, que vivió al poco de mediar el siglo $\mathrm{XV}^{60}$, el Lionel de Limia, que fue primer vizconde de Vilanova de Cerveira y falleció en $1495^{61}$, o también el Lionel de Andrade,

\footnotetext{
${ }^{53}$ Esta doña Ginebra fue dotada por su madre, doña Leonor López de Aguiar, en 4 de julio de 1490 , casando con Ruy Domínguez de Vilouzás, regidor de Betanzos, en quien no alcanzó sucesión, por lo que dejó como heredero de sus bienes, según instrumento otorgado en 2 de mayo de 1531, a su sobrino nieto Ares Conde de Taboada. ẢDM, San Miguel das Penas, leg. 1, núm. 12.

${ }^{54}$ Se la documenta en el testamento de su padre, otorgado en 10 de agosto de 1508. ADM, San Migual das Penas, leg. 1, núm. 85.

${ }^{55}$ Así en el testamento de este personaje, otorgado en 24 de septiembre de 1505 . Véase, Galicia Histórica, Colección Diplomática, doc. 76, pp. 324-347.

${ }^{56}$ Así en el foro que les otorgó a ellos -y ocho voces más- el cabildo de Orense, en 4 de octubre de 1514, de una heredad y una cortiña que fue viña, con sus oliveiras cerca da ponte do Miño, por dieciséis cuartas de vino tinto. E. DURO PEÑA, 1973, doc. 1621, pág. 372.

${ }^{57}$ Este Vasco de Romay otorgó su testamento en 6 de diciembre de 1553, mandando enterrarse en la conventual de Santo Domingo de Pontevedra, en una de las mis sepulturas que allí tengo, que tiene el blasón de mis armas. Aquí también fue enterrada su mujer, doña Ginebra de Araujo, como lo recordaría en su testamento -otorgado en 1592- el hijo de ambos, Teodosio de Romay y Sotomayor, que asimismo ordenó su entierro en aquella iglesia. Noticias en A. SERODIO DOMÍNGUEZ y H. RODRÍGUEZ PÉREZ, El patronazgo de la capilla de Santo Tomás y San Andrés del convento de Santo Domingo de Pontevedra: de los Sotomayor al marqués de Montesacro, "Boletín de Estudios de Genealogía, Heráldica y Nobiliaria de Galicia”, 3 (2004), pp. 288-310. La ascendencia y vínculo de estos Romay en E. PARDO DE GUEVARA, La memoria genealógica y el orgullo de linaje en un instrumento de 1509, "Nobiltà", 70 (2006), pp. 103-127.

${ }^{58}$ Este Lanzarote Mariño, que encabeza la línea de la Casa de Pedreira de Sisán, era hijo del señor de La Sierrạ y repostero de Juan II, Payo Mariño de Lobeira, al que Aponte recuerda como señor de mil quinientos vasallos, que perdió por ser vicioso de mujeres, y de doña Juana Enríquez de Castro, su primera mujer y sobrina del duque don Fadrique de Arjona. El personaje casó con una Sancha Yâñez, en la que hubo a doña Inẹ López de Goyanes, que casó a su vez con Jorge Varela de Dubra, señor de Quindimil, quien testó en 24 de abril de 1569.V. DE APONTE, Recuento, p. 103.

${ }^{59}$ Véase, R. USERo, Sir Lanzarote do Lago e a sua proxenie cedeiresa, Cedeira, 1986.

${ }^{60}$ Hijo de Pedro Vermúdez de Montaos $o$ Vello y de doña Leonor de Castro y Guzmán, su segunda mujer. Este Lionel de Montaos se documentá únicamente por el testamento de su padre, otorgado en 24 de mayo de 1445. Véase, Galicia Diplomática, vol. II, pp. 221 y ss.

${ }^{61}$ El personaje era el segundo de los hijos de Fernán Yáñez de Limia, fallecido en 1422, y de su mujer, la portuguesa doña Inés de Silva Coello. La referencia en E. PARDO DE GUEVARA, Parentesco y nepotismo, p. 70, nota 19.
} 
de filiación y cronología imprecisa ${ }^{62}$. A estos tres testimonios cabría añadir todavía un Leondres López, personaje que se documenta por el año 1496 en la zona de influencia del monasterio de Meira ${ }^{63}$.

Como es sabido, el influjo del nombre de Tristán de Leonois -el perfecto amador de la reina Isolda, tomado como modelo de caballero- fue muy señalado en toda la corona de Castilla. En Galicia, particularmente, su presencia en la onomástica de los linajes de la nobleza fue importante. Es bien conocida, por ejemplo, la sucesión de los Tristán en la estirpe pontevedresa de los Cru y Montenegro, el primero de los cuales se documenta en el tercer cuarto del siglo $\mathrm{XV}^{64}$. Fuera de este grupo familiar, pero también dentro de la ciudad pontevedresa, se documenta por entonces a un Tristán Francés, que ejerció como regidor de la misma por el año $1482^{65}$, mientras que en la Galicia del interior aparece años más tarde un Tristán Enríquez, que actuó como notario público en el coto de San Juan da Lagea, en tierras de Chantada (Lugo), por Ares Pérez Taboada ${ }^{66}$. Frente a ello, el rastro de Isolda es prácticamente inexistente, excepto en su formulación como la reina doña Iseo de la versión de Béroul, que se encuentra en doña Iseo González Taboada, mujer de Juan de Gayoso Noguerol, pese a que no son pocos los documentos que trocan su nombre por el más común de Isabel, fonéticamente próximo ${ }^{67}$, y en doña Iseo Núñez de Berbetoros, documentada ya en el año $1543^{68}$.

${ }^{62} \mathrm{La}$ referencia se toma del foro del lugar de Veiga, en Cerceda, otorgado por la iglesia lucense a este personaje. El extracto documental no lleva fecha, pero atendiendo a su ubicación podría aventurarse su data en los años del segundo pontificado del obispo García Martíz de Vaamonde (1447-1475). Archivo de la Catedral de Lugo, Libro de escrituras, fol. 109v.

${ }^{63}$ Era hijo de un Gonzalo López y de una Constanzạ Gómez, su mujer, constando que por el año mencionado llevaba la granja de Ayaz, propia del citado monasterio. El personaje parece que falleció antes de 1512 . Véase, D. MARIÑO VEIRAS, Señorío de Santa María de Meira (De 1150 a 1525), La Coruña, 1983, p. 347.

${ }^{64}$ Este primer Tristán fue hijo del mercạder y armador Álvaro López [Montenegro?] de Pontevedra, documentado como jurado -o regidor-y procurador del concejo de esta ciudad poco antes de mediar el siglo, y de su mujer, doña Teresa Sánchez de Reino, según se consigna en el tardío epitafio de su sepulcro en Santo Domingo de Pontevedra. Este personaje, que fue muy esforçado y sabio -al decir de Aponte-, se documenta a su vez como arrendador de la sisa de Pontevedra en 1444 y, entre los años 1450 y 1460 , como jurado -en 1454 era alcalde- y, entre los de 1463 y 1473, como juez -en 1470 era tenente de sus torres arzobispales-, constando su muerte violenta a manos de Pedro de Sotomayor, por el año 1479 . Entre la descendencia de este primer Tristán, que había matrimoniado con doña Elvira Prego, hija de Alvaro Barcia, se descubre en la primera mitad del XVI, a un segundo nieto suyo del mismo nombre, como hijo del mercader Pedro Cru y de su mujer, doña Teresa Sánchez, que lo era a su vez de Lope de Montenegro $o$ Vello, que fue muy esforcado y no sabio -también al decir de Aponte- y ejerció como juez de Pontevedra entre los años 1487 y 1498. Véase. J. ARMAS CASTRO, Pontevedra en los siglos XII a XV "Galicia Histórica” (Pontevedra, 1992), pp. 275, 277, 280 y 297. También,
E. FERREIRA PRIEGUE, Galicia en el comercio maritimo medieval, Santiago, 1988, pp. 312, 323 y 333 .

${ }^{65}$ Este personaje casó con Lucrecia Falcón -Falcoa-, documentándose ambos ẹ 6 de agosto de 1482, con motivo del foro del lugar de Matamá, en Tuy, que les concedió el prior y convento de Santo Domingo de Pontevedra. Véase FERNÁNDEZ VILLAAMIL, El tumbillo de Santo Domingo, "Museo de Pontevedra", 4 (1946-1947), pp. 38 y 39.

${ }^{66}$ Así en un instrumento del 7 de septiembre de 1518. ADM, Amarante, leg. 1, núm.

${ }^{67}$ Era hija de Lope de Taboada, señor de la Casa de Taboada, y de doña Sancha Álvarez de Vaamonde, su mujer. La doña Iseo, que otorgó su testamento en 1529, era por tanto hermana entre otros- de Galaor Taboada, señor de Orbán. A comienzos del XVII florecería una nueva doña Iseo de Taboada, hija de Pedro Pardo de Trebolle y de doña María López de Taboada, la cual casó con Ares Conde de Monterroso. Véase lo que se indica en la nota 75.

${ }^{68}$ Así en el foro del lugar de Sistimil. ACL, Tumbo general, fol. 147r. 
Pero este significativo influjo literario en la onomástica de un buen número de linajes de la nobleza local gallega, muchos de ellos vinculados a los puertos comerciales del litoral gallego -Pontevedra, La Coruña o Cedeira, por ejemplo-, no concluye con lo que ha quedado señalado. En este sentido, merece la pena recordar que la materia artúrica, o acaso la troyana propiamente, pero no en cambio la carolingia, ofrece cuanto menos otro testimonio singular: Héctor de Andrade, cuya cronología se sitúa en la segunda mitad del siglo XV, como hijo primogénito de Ruy Díaz de Andrade - era de los Pardo de Cela de Betanzos ${ }^{69}$ - y de su mujer, la venerable vivariense doña Constanza de Castro, señora de Silán, que la tradición beatificó tras descubrirse sus restos incorruptos a comienzos del siglo XVII ${ }^{70}$.

Formando un grupo aparte, pero no por ello de menor interés, se incluirían también en este conjunto los antropónimos procedentes del Amadís, cuyo origen galaico-portugués es motivo de largo debate. En Galicia, de cualquier forma, algunos de aquellos nombres tuvieron una presencia singular. Así, en efecto y muy particularmente, el de Galaor, cuya frecuencia fue destacada hace ya algunos años por I. Beceiro; recuerdo aquí, como ejemplos muy ilustrativos, a los dos Galaor Mosquera, el uno vivió en la primera mitad del siglo $\mathrm{XV}^{71}$ y el otro mientras tanto lo hizo en la segunda ${ }^{72}$, así como a los otros dos Galaor Osorio que vivieron en la segunda mitad de esta centuria, el

\footnotetext{
${ }^{69}$ Este Rui Díaz fue hijo de Lope Núñez de Montenegro y de doña Teresa Rodríguez de Aguiar, su mujer. No puede precisarse el parentesco que unía a los Pardo de Cela con los Andrade, más allá del matrimonio de doña Sancha Núñez -hija de Aras Pardo- con Fernán Pérez de Andrade, uno de cuyos hermanos se había llamado también Lope Núñez de Montenegro, aunque no fue el primero de los varios que se documentan. De cualquier forma, este probable parentesco o simple proximidad es posible que no resulte irrelevante al caso, teniendo en cuenta que Fernán Pérez de Andrade había encargado a su capellán la copia en gallego de la Crónica Troyana. La filiación en Archivo de la Casa de San Pantaleón (Betanzos), leg. 8, núm 89.

${ }^{70}$ El personaje se documenta por vez primera en la carta que Ruy Díaz de Andrade remitió desde el real de Ganada, en 13 de agosto de 1450, a doña Constanzạ, en la que a modo de testamento -se fallecer, esto quede por miña manda-establece que su hijo Héctor herde os bens de San Pantaion, que son de milloria, e a casa e a aldea de Medin, e o señorío que [v]os erdares. La previsión paterna no se materializó finalmente en todos sus términos, pues la Casa de San Pantaleón en concreto pasó a manos de su hermano, Juan López de Andrade de acuerdo con lo que estipularía su madre en el codicilo que otorgó en 2 de diciembre de 1498. İbidem, leg. 8, núm 89 y leg. 10 , núm. 30 .

${ }^{71}$ Nada se sabe sobre su perfil familiar, aunque consta que casó con doña Isabel de Castro, hija tercera de los primeros condes de Lemos, don Pedro Alvarez Osorio y doña Beatriz de Castro. La doña Isabel casó después -probablemente al poco de mediar los cincuenta- con el mariscal don Pedro Pardo de Cela. Archivo General de Simancas, Registro General del Sello, V-1484, fol. 83; la referencia en E. PARDO DE.GUEVARA Y VALDÉS, Los Señores de Galicia, vol. I, pp. 387 y 413. Este personaje debe ser el mismo que se menciona por el año 1439 , como vasallo del rey, en la Crónica del Halconero de Juan II. Véase la edición de J. de Mata Carriazo, Madrid, 1945, Cap. 233, p. 263.

${ }^{72}$ Este Galaor Mosquera, identificado como escudero, casó con doña Ana do Campo, manteniendo los dos una estrecha relación con el monasterio de San Pedro de Rocas, al que defendieron de las incursiones de otros personajes de la nobleza local -en 1490 lo hicieron frente a Juan Pérez de Novoa, Gonzalo de Puga y Payo Sotelo-, recibiendo en recompensa algunos importantes aforamientos entre los años 1472 y el dicho de 1490 . Por lo demás, la trayectoria de este Galaor Mosquera esta muy bien documentada: entre 1473 y 1476 figuraba como alcaide de la fortaleza orensana de Castel Ramiro, mientras que entre 1476 y 1506 figuró ya como regidor de Allariz y merino de la Tierra de Aguiar. Véase, E. DURO PENA, El monasterio de San Pedro de Rocas y su colección documental, Orense, 1972, docs. 264, 307 y 336. También, AHN, Diversos. Serie General, leg. 270.
} 
uno en la proximidad de don Pedro Álvarez Osorio, conde de $\operatorname{Lemos}^{73}$ y el otro como clérigo de la iglesia astorgana ${ }^{74}$, y a Galaor Taboada, cuya trayectoria se documenta ya en los comienzos del XVI ${ }^{75}$. Y junto al recurrente Galaor, cabe destacar también una cierta frecuencia del de Olinda, como, Olinda López de Andrade, documentada en $1481^{76}$, u Olinda Díaz Teixeiro, documentada en 1505 , constando su fallecimiento hacia $1523^{77}$, y Olinda López Noguerol, que ya era difunta en $1532^{78}$. La cronología de todas estas singularidades literarias coincide, por lo demás, con la aparición de otros nombres curiosos entre los hijos bastardos de algunos personajes gallegos de cierto relieve nobiliario. Este es el caso de Galaote - posible diminutivo o deformación de Galaor $^{79}$ _, que lo fue de Sancho Sánchez de Ulloa, primer conde de Monterrey ${ }^{80}$, o también los de Xilo y Arelista, que lo fueron de Ruy Fernández Noguerol, señor de San Miguel das Penas ${ }^{81}$. A este otro tipo de testimonios, que en su momento deberán ser considerados con más detalle, cabe sumar finalmente la aparición de otros nombres también curiosos, aunque de naturaleza probablemente distinta. Menciono como simples

\footnotetext{
${ }^{73}$ No se conoce su filiación; algunos lo han supuesto hijo natural -o bastardo- del primer conde de Lemos, don Pedro Alvarez Osorio, si bien en un documento se le identifica exactamente como primo suyo. Consta muy bien, en cambio, que casó con doña Constanza, hija del mariscal Pardo de Cela y de doña Isabel de Castro, su mujer; este matrimonio parece que fue concertado por el propio conde de Lemos, quien le dio en tenencia las villas de Otero de Rey y Castro de Rey, pero con la condición de que una vez verificado el enlace volvieran a sus manos. El Galaor Osorio, por lo que parece, otorgó su testamento en 13 de mayo de 1480, ordenando su entierro en San Francisco de Astorga. E. PARDO DE GUEVARA Y VALDÉS, Los Señores de Galicia, vol. I, pp. 384 y 415 , y II, p. 198.

${ }^{74}$ Varios criados suyos lo titulan arcediano de El Bierzo al actuar como testigos en un instrumento otorgado en Segovia, en 9 de octubre de 1498. Ibidem, vol. II, págs. 243-245.

${ }^{75} \mathrm{Hijo}$ de Lope de Taboada, señor de la Casa de Taboada, y de doña Sancha Álvarez de Vaamonde, su mujer. Este otro Galaor, que casó con doña Ursula Mosquera, está documentado en la partición de los bienes de sus padres, otorgada en 1509, heredando de ellos el coto de Orbán y otros muchos bienes, entre los cuales figuran varios foros de la encomienda de Portomarín, de la Orden de San Juan. ADM, Amarante, leg. 1 núm. 67, Archivo del Reino de Galicia (en adelante sólo ARG), Pergaminos, núm. 754, y ẢCL, Tumbo general, fol. 110v.

${ }^{76}$ En 17 de noviembre de este año el monasterio de San Pedro de Rocas le otorgó en foro, junto con su marido, Nuño de Amoeiro, ausente, unos bienes en el lugar de Sobrado feligresía de San Miguel de Melias. Véase, E. DUURO PENA, El monasterio de San Pedro de Rocas y su colección documental, doc. 308, pág. 269.

${ }^{77}$ Era hija de Fernán Díaz Teixeiro $o$ Vello y casó con Lope Varela, en el que hubo a un nuevo Fernán Díaz Teixeiro. ARG, Pergaminos, nums. 734 y 849.

${ }^{78}$ Ella era hija de Ruy Fernández Noguerol, señor de Amarante, y de doña Leonor Díaz de Cadórniga, su mujer, que testaron respectivamente en 18 de marzo de 1489 y en 6 de diembre de 1527. Se la documenta, además en el testamento de su hijo, Rodrigo Noguerol, fruto de su matrimonio con Jorge González de Borrajeiros, el cual dejó por heredera a su tía -hermana de su madre- doña Isabel de Noguerol, señora de Amarante. ADM, Amarante, leg. 3 , núm. 6 , y Lemos y Sober, leg. 7, núm. 26. Véanse, también, las notas manuscritas del conde de Canalejas, extraídas del Archivo del Marqués de Figueroa, Mazo de Testamentos, núm. 2, hoy en el archivo de F. de Alós y D. Duque de Estrada.

${ }^{79}$ Esto parece confirmarse en un instrumento de 1479, donde el segundo Galaor Mosquera que quedó mencionado atrás es llamado ahora Galiote Mosquera. Véase, G. F. FERNÁNDEZ SUÁREZ, La nobleza gallega entre los siglos XIV y XV, doc. 36, p. 488.

${ }^{80}$ Otorgado el 24 de abril de 1480 . Véase, BRAG, Colección de documentos históricos, I (1915), doc. 108, pp. 307-316.

${ }^{81}$ Por su testamento, otorgado el 18 de marzo de 1489 , su padre les dejó una manda de 6.000 pares de blancas cada año, al primero, y la presentación de Santa Eugenia de Pallares a la segunda. ADM, Lemos y Sober, leg. 7, num. 27.
} 
ejemplos el Rapote que justo al mediar el XV se documenta como criado de Ares Vázquez de Parga ${ }^{82}$, o el Periches, que años después figuraría entre los escuderos que vivían en el entorno señorial de don Pedro Álvarez de Sotomayor, conde de Camiña ${ }^{83}$.

En términos generales, todas estas modas antroponímicas comenzaron a declinar al avanzar el XVI, coincidiendo con el ocaso del mundo caballeresco y de las ficciones literarias que lo habían recreado y cuya lectura por entonces comenzó a ser considerada como un pasatiempo necio e inútil. La tendencia se agudizó rápidamente al confluir otras circunstancias de signo muy diverso; entre ellas, debieron ser determinantes las propiamente religiosas, que posiblemente puedan explicarse por el ambiente reformador generado en torno al concilio tridentino. Un magnífico testimonio, recordado oportunamente por I. Beceiro, lo ofrece el sínodo orensano del 22 de abril de 1544, donde el obispo Francisco Manrique de Lara ordenó a los clérigos de su diócesis la imposición de los nombres de sanctos e sanctas que están en el cielo, porque se les déis por abogados, e no Héctor ni Roldán, ni otros que havéis acostumbrado a les poner ${ }^{44}$. Y sin duda esta instrucción no fue única.

Fecha de recepción del artículo: octubre 2008.

Fecha de aceptación y versión final: marzo 2009.

\footnotetext{
${ }^{82}$ Por su testamento, otorgado el 11 de agosto de 1450 (se trata de una copia de veracidad no bien contrastada), el de Parga le dejó una capa y su quiñón de Sanjurjo, hasta que su hijo Vasco fuera mayor de edad. ADM, Parga, leg. 1, núm. 96.

${ }^{83} \mathrm{El}$ personaje actuó como testigo en la escrituración de su testamento, en 15 de diciembre de 1476. Véase, V. LISQUIBEN, Copia de uno de los testamentos de Pedro Madruga, "Boletín de la Comisión de Monumentos de Orense", 17 (1949), pp. 131-135.

${ }^{84}$ Véase, Constituciones sinodales del obispado de Orense de 1544 (facsímil del Consello da Cultura Galega, Lugo, 1994), Fol. Vv. El texto reproducido en Synodicom Hispanum, vol I. Galicia, edicion de A. García García, Madrid, BAC, 1981, pág. 153.
} 